\title{
doispontos:
}

\section{Tarde avant Bergson Le statique et le dynamique}

\author{
Pierre Montebello \\ Université de Toulouse-le-Mirail, Toulouse, France \\ pierremontebello56@gmail.com
}

Resumo: Retraçar influências é importante, mas a relação entre dois pensadores não pode ser somente a de uma influência, pois toda influência é retrospectiva. De que se trata aqui é, sobretudo, da retomada de um gesto especulativo que engaja e se prolonga. Os princípios metafísicos de Tarde são de tal magnitude que, ao lê-los, não podemos mais separar Bergson de uma poderosa visão que começa antes dele. Com a intenção de flagar a continuidade de um mesmo gesto de pensamento entre esses dois autores, iremos abordar, neste texto, o psicomorfismo de Tarde, sua suposição de um divisão na natureza e algumas das consequências sobre a moral e a religião passíveis de serem dele retiradas e que, acreditamos, possuem alguma concordância com o bergsonismo.

Palavras-chave: Bergson; Tarde; Natureza; Psicologia; Sociologia

\section{Tarde before Bergson}

\section{Static and dynamic}

\begin{abstract}
Tracing influences is important, but the relationship between two thinkers cannot be just an influence. Because, all influence is retrospective. We are rather dealing with the resumption of a speculative gesture that engages and continues. Tarde's metaphysical partisanship is so considerable that by reading it one can no longer detach Bergson from a striking vision that begins below him. With the pretension of grasping the continuity of the same gesture of thought between these two authors, we will approach, in the present text, the psychomorphism of Tarde, with his assumption of a division of nature and some of its consequences on Morality and mysticism, in what seems to us, they are not without consonance with Bergsonism.
\end{abstract}

Keywords: Bergson; Tarde; nature; psychology, sociology

Bergson et Tarde sont liés académiquement par un curieux concours de circonstances. Tarde obtient la chaire de Philosophie Moderne du Collège de France en 1900 après l'avoir refusée en 1899, parce qu'il souhaitait qu'elle soit transformée en chaire de sociologie. Bergson a écrit de son côté à l'administrateur du Collège de France pour obtenir cette chaire. Il est déçu qu’elle soit attribuée à Tarde : « Je n’ai pas de chance, écrit-il à Mme Rafflalovitch, c'est $M$. Tarde qui est nommé ( ... ) J'avoue que j’ai éprouvé une assez vive déception ». Tarde meurt en 1904, le 12 mai. Le 13 novembre, l'assemblée des professeurs du Collège de France décide de maintenir la chaire de Philosophie moderne et de nommer Bergson sur ce poste. Le 19 novembre un décret nomme Bergson « en remplacement de Monsieur Tarde, décédé ». C’est donc dans le sillage direct de Tarde que Bergson entre dans cette prestigieuse institution. 
Mais Bergson connaît-il bien la philosophie de Tarde ? En 1903, Bergson prend part à une discussion sur la philosophie de Cournot, plus précisément sur la thèse que Tarde lui consacre « La philosophie sociale de Cournot $\gg$. Dans ce texte, Tarde expose la conception singulière que Cournot se fait de l'histoire : il distingue, selon Tarde, un état pré-historique des sociétés, quand celles-ci sont sous « l'impulsion et la direction souveraine de l'instinct vital $»$, et les sociétés historiques « lorsque l'importance grandissante des individualités exceptionnelles et des circonstances fortuites leur permet de se mesurer avec la Raison, avec la force des choses, et d'engager avec elles un long combat aux nombreuses péripéties qui font la beauté du drame humain ». (Apud, BERGSON, 1972, p. 589)

Il y a chez Cournot déjà, selon Tarde, une recherche sur ce qui distingue sociétés vitales et sociétés historiques. Cournot s'appuie sur une distinction qu' il juge cruciale : cause et raison. La cause est productive, factuelle, elle rend compte des faits historiques. Mais la raison est explicative, supérieure, mathématique, parce qu'elle met en rapport les faits et justifie leur développement. Selon Tarde, toutefois, elle conduit aussi la sociologie à prendre pour réalité des fantasmagories et des illusions. Ainsi, mettre en rapport deux séries numériques, celle des suicides et celle de la température durant une année, donne l'illusion que l'on a expliqué le suicide alors que l'on ne comprend rien des forces internes et toujours spéciales qui sont en jeu ici. Un tel schéma conduit à une « explication transcendante des sociétés ». Bergson en posent la question de savoir s'il a bien compris la distinction entre raison et cause montre que la pensée de Cournot ne lui est pas familière. En 1915, il fait un éloge appuyé de la méthode critique de Cournot («Il est temps de mettre ce penseur à sa vraie place -une des premières- parmi les philosophes du XIXème siècle »), qu'il a lu dans sa jeunesse. Mais, il est plus proche de la psychologie inter-mentale qui est selon Tarde le foyer vif de la sociologie. Le moteur de l'histoire ne se saisit pas par la raison (la direction générale d'un cours d'eau) mais par la multitude d'influences des agents (l'action des molécules).

Bergson a laissé, en sus de cette discussion, deux témoignages plus importants de son estime pour Tarde. Dans un texte en 1909, une préface écrite pour un recueil de «Pages choisies » de Tarde, Bergson affirme que le grand mérite de Tarde est d'avoir en effet donné « une sociologie nettement psychologique, et une psychologie toute prête à s'épanouir en sociologie » (BERGSON, 1972, p. 811). Nous allons vérifier dans un instant, la signification de la deuxième partie de l'assertion, c'est-à-dire la transition du psychique au social. Tarde opère selon lui la synthèse entre individuel et social avec une «puissance d'imagination » sans commune mesure. Notons que Bergson semble fasciné par «l'imagination » de Tarde. C'est cette puissance d'imaginer qui se trouve au centre des deux textes qu'il écrit sur Tarde.

Mais, que recouvre en vérité ce terme ? Il désigne certes l'invention philosophique, la vaste curiosité d'esprit, l'ingéniosité du penseur qui fourmille d'idées nouvelles, apporte « d'ingénieuses solutions », la capacité à tout brasser et à tout embrasser. Mais peut-être plus que cela encore. Peu de temps avant la rédaction de cette préface, toujours en 1909, le 12 septembre, Bergson prononce un discours sur Gabriel Tarde, qui est en fait un profond éloge de sa manière de faire philosophique. Bergson s'y dit frappé « par la fantaisie » de Tarde, ses vues « originales, brillantes », innovantes, multiples, imprévisibles. Il retient des lois de l'imitation, que l'évolution des sociétés suppose toujours des actes singuliers, des initiatives individuelles, il y apprend « comment un simple geste individuel, tombant dans le milieu social comme une pierre dans l'eau d'un bassin, l'ébranle tout entier par des ondes imitatives qui vont toujours s'élargissant », nous donnant ainsi un « sentiment aigu de notre responsabilité ». Bergson conclut : « Saluons en Tarde le philosophe à la pensée pénétrante, à l'imagination hardie, qui nous a ouvert tant d'horizons »(BERGSON, 1972, p. 799-801).

Qu'exprime au fond cette « imagination hardie » de Tarde aux yeux de Bergson ? Essentiellement, une formidable capacité spéculative. C'est-à-dire une manière de tracer des univers nouveaux, au sens littéral, 
de faire monde, de faire communiquer les choses pour tracer un monde. « A travers tant de réflexions fines, tant de réponses ingénieuses à tant de questions élégamment posées, c'est une certaine conception générale de la réalité, c'est derrière cette conception elle-même, une certaine vision simple qui transparait... », écrit Bergson dans sa préface aux « Pages choisies » de Gabriel Tarde (BERGSON, 1972, p.812). Bergson n'ignore pas que Tarde appuie toute ses considérations sociales sur « certaines vues métaphysiques profondes sur la nature de l'univers, des éléments qui le composent et des actions que ces éléments exercent les uns sur les autres $\gg($ BERGSON, 1972, p.800).

N'est-ce pas précisément cette puissance spéculative capable d'ouvrir des horizons nouveaux dont on trouve encore la trace, ou l'imitation rayonnante, dans Les deux sources de la morale et de la religion (BERGSON, 1932) ? Nous ne pouvons que suivre Jean Milet lorsqu' il dit dans sa thèse sur Tarde, Gabriel Tarde et la philosophie de l'histoire, qu' il y a cinq thèmes philosophiques, où l'influence de tarde sur Bergson paraît manifeste:

«Tarde a été le premier en son temps, autant qu'il paraisse à soulever le problème des rapports en le Statique et
le Mouvant, et à opter résolument pour la priorité du Mouvant (...). En second lieu, Tarde a été aussi un des
premiers à remettre publiquement à l'ordre du jour le problème de la signification épistémologique du Calcul
infinitésimal (...). Autre point de convergence : Tarde a formulé une théorie de l'Evolution créatrice qui annonce
nettement celle de Bergson. Il invoque un Elan vital, et il accrédite en même temps, l'idée d'une Evolution qui serait
en elle-même créatrice. L'influence de Tarde sur Bergson est encore plus sensible dans le domaine des questions
morales et religieuses. Tarde fournit à Bergson une analyse de la causalité exemplaire (à laquelle Bergson se réfère
explicitement dans sa lettre de 1909) qui sera mise à profit par Bergson dans les deux sources. Chez Tarde, les Héros
et les Génies n'imposent pas leur volonté, ils sollicitent par l'exemple; or, il en sera exactement de même pour les
Héros et les Mystiques, chez Bergson. Nous trouvons même chez Tarde une théorie des « deux sources »e la
vie morale : morale de la « contrainte » et morale de « l'inspiration », cette théorie réapparaîtra, presque dans les
mêmes termes, chez Bergson en 1932. Enfin, cinquième et dernier point, et sans doute le plus important, Tarde a pu
fournir à Bergson une doctrine, déjà très élaborée, sur la signification métaphysique et théologique de l'expérience
des mystiques. Les mystiques ne sont pas des malades, ce sont des témoins véridiques. Il faut les regarder comme
les messagers des Réalités supérieures. Tarde est conduit, de là, à l'hypothèse de Réalités supérieures. Bergson va
jusquà la reconnaissance d'un Dieu personnel. Les conclusions sont différentes ; mais les points de départ ont été
les mêmes. Tarde avait ouvert la voie; Bergson s'y est engagé, et il est allé plus loin » (MILET, 1970, p.387-388).

Ce tableau pose bien des questions d'histoire de la philosophie. Retracer des influences est important, mais la relation entre deux penseurs ne peut être seulement une influence. Car, toute influence est rétrospective. On a plutôt affaire à la reprise d'un geste spéculatif qui engage et se prolonge. Les partis-pris métaphysiques tardiens sont considérables au point qu'en le lisant qu'on ne peut plus détacher Bergson d'une saisissante vision qui commence en-deçà de lui. Il n'est pas possible de revenir sur chacun de ces cinq points présentés par Milet. L'essentiel nous semble de saisir d'abord le psychomorphisme de Tarde qui suppose une division de la nature qui n'est pas sans consonance avec le bergsonisme, enfin de nous attarder sur quelques aspects de la morale et du mysticisme.

L'aspect le plus connu de la philosophie de Tarde est son psychomorphisme, le fait de ramener toute action dans la nature à une quantification du désir et de la croyance. Tarde développe, dans son premier génial article sur ce sujet ( «Le désir et la croyance, la possibilité de leur mesure »), avec l'idée que tous nos états d'âme se ramène au fond au sentir, à la croyance et au désir, qui rendent compte des modes d'agrégation, de composition, d'association ou de dissociation de tous les éléments psychiques sensoriels. Tarde donne pour la première fois au temps et à l'espace une dimension clairement psychique. L'espace et le temps ne sont pas des cadres, ce sont des opérations du désir et de la croyance, à tous les niveaux monadologiques du réel. L'espace, qu'est-ce d'autre en effet que l'objectivation de la faculté de coordonner, de discerner, d'établir des coexistences et des systèmes, d'agréger, de lier, et donc de croire ? Le temps lui est la transposition objective de l'effort, du désir, de la captation élémentaire, de la volonté qui cherche à se propager, « désir 
universel » qui sous-tend l'univers, qui fait que chaque atome aspire à être univers. Bien avant Bergson, Tarde peut donc écrire. « L'espace, n'est-ce pas l'intelligible par excellence, la compatibilité et la séparabilité universelles, l'assimilabilité et la discernabilité infinie, autant dire l'affirmabilité et la niabilité de toutes choses, leur crédibilité, et en même temps chose remarquable, leur mesurabilité essentielle ? » (TARDE, 1999a, p.199-200). Dans l'espace, statique, statistique, agrégatif, synthétique, on mesure des forces de croyance, on quantifie des adhésions moléculaires, on nombre des effets de masse, des équilibres sédentaires. Il n'y a donc pas d'espace en soi, mais un espacement continu, une intégration micrologique, une contamination physique, vitale, psychique et sociale, une foi qui rassemble. L'ondulation physique, la reproduction vitale, l'imitation sociale ne sont pas dans l'espace, ce sont des modes d'espacement qui reposent sur la répétition, l’adhésion, la répétition. Et de même, le temps unique n'existe pas, le temps se dissémine en multitudes de désirs élémentaires, en foyers de forces incompressibles qui forment les dynamismes de l'univers, les lignes temporelles qui le sillonne : « Le temps, n'est-ce pas la Volonté pure, sans but, mais susceptible de tous les buts, l'optatif catégorique, vouloir virtuel de tous les vouloirs » (Ibid., p.200).

Tarde introduit le psychomorphisme en philosophie contre l'anthropomorphisme, notons-le bien. Il y a nécessité à supposer que les forces qui sont en nous sont aussi dans tous les échelons de l'univers. Oui, la nature, l'univers matériel, sont esprit, à condition de bien voir ce que l'on dit. Il ne s'agit pas d'affirmer que l'univers «se compose de mes états d'esprits » mais de dire que tous les éléments de l'univers sont semblables à nos états d'esprit. Schopenhauer procède d'ailleurs à cette même analogie qui va de notre volonté à la nature. C'est ainsi que pour Tarde l'on peut tenter la « spiritualisation de l'univers », en montrant que le psychomorphisme traverse la nature, de la vie humaine à l'animal, de la molécule à l'atome. La nature semble dès lors se diviser en deux tendances qui vont avoir une importance assez considérable chez Bergson : le statique et le dynamique, « la force statique appelée croyance et la force dynamique appelée désir » (TARDE, 1999b, p. 48). C'est dans les Lois de l'imitation que Tarde met en scène le plus nettement cette dualité si cruciale pour Bergson, entre plasticité et fonctionnalité, organisation et dynamisme créateur, effets de masse et activité possessive, la tendance des monades à se rassembler et leur tendance à essaimer, se propager, capter, posséder (on sait la formule célèbre de Tarde, substituer une métaphysique de l'avoir à celle de l'être car « la possession est le fait universel »):

«L'invention et l'imitation sont l'acte social élémentaire, nous le savons. Mais quelle est la substance ou la force sociale dont cet acte est fait ; dont il n'est que la forme ? En d'autres termes, qu'est-ce qui est inventé ou imité ? Ce qui est inventé ou imité, ce qui est imité, c'est toujours une idée ou un vouloir, un jugement ou un dessein, où s'exprime une certaine dose de croyance et de désir, qui est en effet toute lâme des mots d'une langue, des prières d'une religion, des administrations d'un État, des articles d'un code, des devoirs d'une morale, des travaux d'une industrie, des procédés d'un art. La croyance et le désir : voilà donc la substance et la force, voilà aussi les deux quantités psychologiques que l'analyse retrouve au fond de toutes les qualités sensationnelles avec lesquelles elles se combinent; et lorsque l'invention, puis l'imitation, s'en emparent pour les organiser et les employer, ce sont là, pareillement, les vraies quantités sociales. C'est par des accords ou des oppositions de croyances s'entre-fortifiant ou s'entre-limitant, que les sociétés s'organisent; leurs institutions sont surtout cela. C'est par des concours ou des concurrences de désirs, de besoins, que les sociétés fonctionnent. Les croyances, religieuses et morales principalement, mais aussi juridiques, politiques, linguistiques même (car, que d'actes de foi impliqués dans le moindre discours, et quelle puissance de persuasion, aussi irrésistible qu'inconsciente, possède sur nous notre langue maternelle, vraiment maternelle en cela !), sont les forces plastiques des sociétés. Les besoins, économiques ou esthétiques, sont leurs forces fonctionnelles.

Ces croyances et ces besoins, que l'invention et l'imitation spécifient et qu'en ce sens elles créent, mais qui virtuellement préexistent à leur action, ont leur source profonde au-dessous du monde social, dans le monde vivant. C'est ainsi que les forces plastiques et les forces fonctionnelles de la vie, spécifiées, employées par la génération, ont leur source au-dessous du monde vivant, dans le monde physique, et que les forces moléculaires et les forces motrices de celui-ci, régies par l'ondulation, ont aussi leur source, insondable à nos physiciens, dans un monde hypophysique que les uns nomment Noumènes, les autres Énergie, les autres Inconnaissable. Énergie est le nom le plus répandu de ce mystère. Par ce terme unique on désigne une réalité qui, comme on le voit, est toujours double en ses manifestations; et cette bifurcation éternelle, qui se reproduit sous des métamorphoses surprenantes 
à chacun des étages superposés de la vie universelle, n'est pas le moindre des traits communs à signaler entre eux. Sous les appellations diverses de matière et de mouvement, d'organes et de fonctions, d'institutions et de progrès, cette grande distinction du statique et du dynamique, où rentre aussi celle de l'Espace et du Temps, partage en deux l'univers entier. » (TARDE, 1993, p. 159).

Ce division du réel, « cette réalité double en ses manifestions », cette « grande distinction du statique et du dynamique », « la bifurcation éternelle », est bien sûr capitale. Mais d'abord parce qu'elle est un trait structurel de l'époque. On la trouve chez Schopenhauer (Volonté et représentation), chez Nietzsche (Dionysos et Apollon), puis chez Tarde, Ravaisson et Bergson. La philosophie change de cap. Elle n'est plus hypnotisée par le dualisme du corps ou de l'esprit, par la question du cogito. Elle devient philosophie de la nature, cosmologie, tentative de trouver un schéma moniste général qui, à partir des forces qui nous traversent, prétend embrasser toutes les strates de la nature. C'est alors l'ensemble du réel ou de la nature qui se polarise entre création et conservation, espace et temps, dynamisme gestatif et repliement statique, acte et automatisme, énergie créatrice et stabilisation, hétérogène et homogène.

L’œuvre de Ravaisson manifeste une telle dualité. On sait que Bergson y a été très sensible. L'éloge de Ravaisson - « La vie et l'œuvre de Ravaisson »-, à la toute fin de La pensée et le mouvant, ne doit pas s'entendre en un autre sens que celui de Tarde. Dans De l'habitude, nous dit Bergson, « c'est toute une philosophie de la nature » qui est en jeu (BERGSON, 2009, p. 266). Bergson est très intéressé par le fait que, chez Ravaisson, la nature soit expliquée par une « expérience intérieure », à savoir l'habitude qui fait passer par degrés insensibles l'acte libre en automatismes, et retomber la conscience en inconscience (Idem, p. 267). La nature ne serait-elle pas de même « une conscience obscurcie et une volonté endormie », et la matière un « assoupissement de l'esprit » (Idem, p. 267 et 275)? Cette solution revient à ne plus opposer esprit et nature, sans les identifier pour autant, et surtout elle permet de s'affranchir d'un strict mécanisme qui réduit tout à la matière. Les thèses de Ravaisson ont « frappé les contemporains » parce qu'elles allaient à rebours du physicalisme dominant (Idem, p. 267). Dans l'analyse physique de la nature, on prétend en effet passer de la matière à la vie, puis à la conscience, comme si les causes matérielles ou physiques étaient premières, comme si l'on pouvait restituer la chaîne des êtres vivants, puis des êtres conscients, en détaillant toujours plus précisément l'enchaînement de ces conditions matérielles. Ravaisson propose de suivre le chemin inverse qui va d'une plus haute activité spirituelle à la matière. La nature est une activité spirituelle divine qui incline progressivement vers automatisme et mécanisme, par répétition de son acte libre. Quoi de plus étonnant, dit Bergson, pour ceux qui en restent au physicalisme, que cette inversion de perspective qui explique le bas par le haut, et non l'inverse : « Quoi de plus hardi, de plus nouveau, que de venir dire aux physiciens que l'inerte s'expliquera par le vivant, aux biologistes que la vie s'expliquera par la pensée », aux esthètes que la beauté est la grâce du mouvement intime des choses, aux astronomes que l'univers se manifeste par un acte de « condescendance » divin qui est son origine (Idem, p. 291)?

Même admiration de la hardiesse, notons-le, chez Tarde et Ravaisson. C'est elle qui donne à Bergson l'impulsion pour établir que la spiritualité n'est pas bornée à l'âme ou à Dieu mais s'étend à tout l'univers, à toutes les réalités, qu'elle traverse l'univers, soit comme psychomorphisme (Tarde), soit comme activité spirituelle qui retombe (Ravaisson), soit comme durée créatrice qui se spatialise. Le vrai spiritualisme est un spiritualisme non de l'esprit, mais de la nature, soulignons-le. C'est ainsi que le spiritualisme s'accomplit, lorsqu'il finit par se loger dans le cœur des choses, et non lorsqu'il s'en sépare. De ce mouvement, de ce réalisme spiritualiste, Tarde et Ravaisson sont deux exemples frappants

Venons-en alors aux jonctions tout à fait étonnantes avec les Deux sources (BERGSON, 1932). Tarde a été amené dans sa Logique sociale, en s’appuyant sur cette infra-logique du désir et de la croyance, à réfléchir sur les formes de socialité et d'histoire. « Les nations closes », écrit Tarde commencent par se hair avant de se 
connaître. Il importe de faire une histoire du patriotisme et de la haine, pour mieux saisir en retour l'effusion sympathique, la propagation des sentiments qui nous font excéder cette clôture, tels les sentiments religieux chrétiens sous leur forme esthétique ou religieuse. Tarde est le premier à décrire deux systèmes de moralité:

\begin{abstract}
« N'est-il pas vrai qu'il y a deux genres de moralité ? L'un, propre aux peuples barbares et aussi aux incomplètement civilisés, dérive d'un principe tout patriotique, c'est-à-dire politique et militaire, auquel la conduite entière est soumise, comme à Sparte et dans la Rome des premiers temps. Courage, sobriété, discipline, obéissance aux chefs : voilà les vertus cardinales. L'autre, - dont le germe s'entrevoit dès les temps les plus reculés dans certaines prescriptions telles que le devoir de l'hospitalité et même la vendetta, mais ne se développe quà la longue, - procède d'une inspiration au fond esthétique, quoique d'abord présentée sous couleur religieuse. Le salut chrétien, chez les âmes les plus élevées du christianisme, n'est-il pas une contemplation désintéressée de la beauté divine ? Même la grâce idéale des houris est, chez les musulmans, un attrait sublime ; et l'honnêteté, la conformité aux lois, nullement utilitaires, de l'honneur, est certainement, pour «l'honnête homme » du XVIIe siècle, un beau nouveau à réaliser en soi. Pendant que la science élucide le vrai, l'art, sous les espèces du beau, collabore à la création du bien, le précise, le personnifie, le vivifie (TARDE, 1999c, p. 597).»
\end{abstract}

La justification de la guerre, « le militarisme nécessaire comme l’ontologisme social », ainsi que toutes les théories fondées surl'égoïsme, la concurrence vitale, économique, étatique (Tarde se prononce contre Darwin et le darwinisme politique, le sens de l'évolution n'est pas la lutte mais la différenciation multilinéaire interne qui se poursuit en harmonisations cohérentes et progressives) sont incompréhensibles aux yeux de Tarde, d'autant qu'on a souvent coutume d'y voir le fin mot de l'histoire. L'argument ultime est de justifier la guerre par l'agir libre qui rencontre la résistance d'autrui. Mais, être libre ne signifie pas faire triompher son moi contre les autres :

« Liberté signifie essentiellement non pas lutte, mais diversité, originalité, caractère. Et c'est dans les voix de la paix et de l'association, non sur les champs de bataille, que les originalité s'accentuent, que les aptitudes spéciales, caractérisées, s'utilisent et se développent réciproquement (TARDE, 1999c, p.369)».

Certes, on ne peut nier que l'ambition propagatrice de tous les éléments de la nature se traduit dans la sphère humaine, par la lutte nécessaire contre la nature, contre les autres vivants, par exemple, la chasse, le conflit entre tribus, entre nations... Mais, l'humanité naissante n'a pas su non plus faire un choix entre deux formes de culture :

\begin{abstract}
« Ces deux chemins étaient : la culture à outrance de l'égoïsme par la guerre, l’anthropophagie, l'esclavage, le despotisme asiatique et la culture à outrance de la sympathie par la religion, le droit, le commerce, la science, la morale, l'art. Elle n’a pas choisi entre les deux, elle a pris tantôt l'un, tantôt l'autre, les unissant et les coupant par des chemins de traverse. Elle a ainsi masqué sous des institutions adultères, sous des compromis sacerdotaux et superstitieux, ou guerriers et chevaleresque, la divergence radicale de ces deux carrières, et elle a faussé le sentiment religieux, qui est au fond, essentiellement, non la peur, mais l'amour, l'ouverture du cœur, le besoin d'étendre sans cesse le champ social et la sympathie du cœur (Idem, p. 370)».
\end{abstract}

Comme cela est visible, «nations closes » et « ouverture du cœur » forment déjà une opposition chez Tarde, expressément. Plus encore, les pages sur la guerre à la fin des Deux sources consonent avec celles de Tarde. « La nature a-t-elle voulu la guerre ? Répétons une fois de plus, que la nature n'a rien voulu », écrit Bergson, et cela même si l'intelligence fabricatrice dispose au fond l'homme à lutter contre la matière et les autres. Reste que l'instinct de guerre, originel, ne peut être déraciné, même dans la société ouverte, il peut seulement être retourné ou « réprimé » par une autre tendance à laquelle Bergson rattache le judéo-christianisme et l'amour. Même constat de Tarde qui se demande si l'on peut extirper totalement la guerre, même horreur des deux devant la guerre moderne, sa monstruosité ( $\ll$ les massacres sont d'une horreur que les grecs n'auraient même pas imaginée » Bergson), même conviction d'une seconde voie pour la morale et la religion, d'une autre ouverture. C'est sans doute le contexte nationaliste et guerrier qui explique à la fois L'opposition universelle de Tarde (1897) et les Deux sources de Bergson (1932), même si ces textes sont séparés par de nombreuses années. Ils bornent en fait la lente maturation des deux guerres mondiales : « Les nations, à cette heure, s'arment jusqu'aux dents pour la guerre des nations, les classes pour la guerre des classes ; et la paix armée des classes, prélude, dit-on, de la catastrophe sociale qui s’annonce, 
est encore plus effrayante que la paix armée des Etats. Est-elle inévitable pourtant, cette lutte fratricide et inexpiable, le plus colossal des fratricides que la terre aura jamais vus (TARDE, 1999a, p.374)? »Ce pessimisme fait écrire à Tarde, en 1892, un conte assez fantaisiste, Les Géants chauves : Les chauves sont déçus par la calamiteuse gestion politique des chevelus, ils se révoltent, prennent le pouvoir, mettent en place un pouvoir plus aberrant encore, si bien que l'humanité périt dans un massacre général.

Dans ce cadre là, il va de soi que c'est l'exemple qui est décisif pour nous orienter d'un côté à l'autre, du clos à l'ouvert. Bergson a, on l'a vu, soulignél'extrême responsabilité en quoi consiste justement l'exemplarité pour nous faire passer du clos, de la logique guerrière, à l'ouvert. Mais ne parlons pas des génies et des héros, parlons des mystiques que Les deux sources mettent en scène. C'est à Tarde qu' il revient, après Schopenhauer, de leur donner une place irremplaçable. Car, si la religion est « œuvre imitative » au premier chef, et donc sympathie universelle, le mysticisme est son expérience la plus cruciale. « Les mystiques, ces hautes futaies de la forêt religieuse, ont été clairvoyants en ceci. C'est une chose véritablement frappante que la ressemblance de tous ces inspirés à toute époque et en toute religion. Je ne parle pas de l'extase proprement dite, qui, en tant que névrose, est la même toujours, précipice pathologique côtoyant cette ascension, comme la folie le génie, mais où tous ne sont point tombés. Qưon lise Plotin ou Sainte-Thérèse, Marc-Aurèle ou Fénelon, on les verra s'accorder avec les mystiques hindous dans la description de leurs états intimes, de leur douceur souveraine goûtée à ne plus vouloir, à se laisser conduire par l'hôte divin, à se remplir d'une contemplation, d'une conviction stable, absolue, et absolument sans trouble de désir. Le nirvãna en son meilleur sens, n'est pas autre chose. Les mystiques de l'Inde distinguent, comme Sainte-Thérèse, divers degrés de l'état d'oraison, qui ont reçu des noms différents. Le point culminant est l'yoga, «l>union où l>âme sent qu>elle est en Dieu et que Dieu est en elle ${ }^{1} \gg$. Est-ce donc tout à fait une illusion, cette foi commune à tant de grands esprits qui ne se connaissent pas et qui l>ont puisée au for intérieur ? Ne dirait-on pas plutôt qu>en cette expérience intime $s>$ est déchirée à leurs yeux l>erreur des nôtres, l'illusion de notre autonomie absolue et de notre réalité radicale, qui nous empêche de sentir en nous l'action de la virtualité infinie, de l'universelle puissance ? Quoi qu'il en soit, d'ailleurs, le sentiment illusoire ou non de cette copossession du moi par son non-moi intime, qu'il appelle son Dieu, et réciproquement, est la source d'un étrange amour qui rejaillit souvent sur l'universalité des créatures. Avec quel lyrisme Plotin parle de ce Dieu du cœur ! « Sans l'avoir vu encore, on le désire comme le bien, et, quand on le voit, on le contemple comme le beau, on est rempli de frémissement et de joie, on est frappé d'un effroi qui ne trouble pas, on aime d'un véritable amour, on a d'âpres désirs, on sourit de pitié pour les autres amours, et on dédaigne les choses que jadis on croyait si belles (TARDE, 1999c, p.396-397)». On dirait une page de Sainte-Thérèse.

Est-ce que ce ne sont pas les mêmes mots ou presque, qui reviennent sous la plume de Bergson ? D’abord la séparation de l'expérience mystique et de la crise pathologique : « On pourra bien parodier le mysticisme et il y aura une folie mystique, suivra-t-il de là que le mysticisme soit folie ? » (BERGSON, 1932, p.242). « Quand on prend à son terme l'évolution mystique, on se demande comment ils ont pu être assimilés à des malades $\gg$ (Idem, p. 241). Ensuite, «l'universalité » de l'expérience mystique, qui établit un pont entre toutes les religions : « Il faut tout d'abord remarquer l'accord des mystiques entre eux » (Idem, p.261). Puis, ce retour à la nature naturante, au « principe de la nature », d'où résulte « un tout autre attachement à la vie », une « confiance transfigurée » (Idem, p.227). «A nos yeux le mysticisme est une prise de contact, et par conséquent une coïncidence partielle, avec l'effort créateur que manifeste la vie. Cet effort est de Dieu, si ce n'est pas Dieu lui-même $\gg$ (Idem, p. 233). Enfin, l'union mystique, qui consiste à replacer son vouloir en Dieu, et d'où résulte l'exaltation ou l'amour qui se répand sur tout le créé, sur toutes les créatures.

Voila bien des points communs qui renvoient aussi à Schopenhauer, le premier à faire de l'expérience mystique une expérience cosmologique. Pour finir, disons clairement que les thèses de Tarde sont des 
thèses humanistes. Celles-ci sont nées dans le contexte des écrits d'Alberti, elles travaillent l'occident en silence. ${ }^{2}$ La religion comme souffle humanitaire ; l'art en tant que « joie sociale $\gg$ ou harmonie qui égalise les différences en les respectant, qui dépasse les nations, « supranational » en un mot; le beau et le bien comme moteurs de l'évolution historique ; l'éloge de «l'invention $\gg$ individuelle (terme essentiel d'Alberti) et du consensus social. Autant de thèses humanistes. ${ }^{3}$ Selon Tarde, l'harmonisation sociale est favorisée par la religion et l'art. Au fond, ces domaines sont comme des loupes qui permettent de saisir la force de l'individuel au travers de l'action des mystiques et des artistes. L'amplification historique, l'exemplarité, le rayonnement imitatif, conduiront nécessairement à une civilisation unique sans patries, ni nations, ni guerre, seulement mue par une différenciation et une inégalité interne qui n'est autre que la rivalité nécessaire au progrès. Bergson n'est pas loin de ces thèses humanistes, de cet humanisme cosmologique, de ce cosmos humanisé. Tarde aura ainsi été un exemple pour Bergson prouvant la valeur même des lois rayonnantes de l'imitation. Laissons le dernier mot à Bergson:

\begin{abstract}
« Des éléments analogues par certains côtés aux monades de Leibniz, mais, à la différence des monades leibniziennes, capables de se modifier les unes les autres, voilà ce que Tarde met au fond de la réalité. Divers dès le début, ils accentuent leur diversité sans cesse, grâce à l'action qu'exerce sur eux leur entourage, grâce à l'action qu'ils exercent sur eux-mêmes. Ils composent ainsi une société où chacun développe sa propre individualité, et par une espèce de rayonnement, l'individualité des autres. Ils forment un univers, auquel le jeu de plus en plus varié des initiatives, qui s'accordent de mieux en mieux entre elles, donne de plus en plus l'aspect d'une œuvre d'art » (BERGSON, 1972, p. 812).
\end{abstract}

Peut-être ce cosmos humanisée est-il ce qui nous intéresse le moins chez Tarde et Bergson aujourd'hui. Mais, pour lui donner quelque consistance, il aura fallu replacer l'homme dans le cosmos, en quelque sorte désanthropologiser l'homme, et c'est finalement ce geste capital qui ouvrira à une métaphysique de la nature moderne et à des lectures comme celle de Simondon et Deleuze.

\title{
NOTAS
}

1. Barth, les religions de l'Inde, cité par Tarde.

2. Voir, Bertrand Prévost, L'humaniste, le peintre et le philosophe. Théorie de l'art autour de Leon Battista Alberti, sous presse.

3. Tarde, La logique sociale, p. 589 : «L'art est un puissant moyen d'accorder les désirs et les croyances. Il accorde les désirs, non, comme l'industrie et le commerce, en faisant servir chacun d'eux aux fins des autres, sans diminuer en rien leur dissemblance : il les identifie, il les unit en faisceau, en une grandiose union, come fait l'action guerrière. Mais, tandis que la guerre obtient la convergence des désirs nationaux au prix d'un conflit international, l'art, alors même qu'il est un simulacre de combats, concourt à l'union fédérative des peuples voisins, en suscitant un objet supranational, un beau nouveau, prompt à franchir les frontières les plus hautes. L'art est le grand magicien, le grand charmeur des serpents d'âme ».

\section{REFERÊNCIAS}

BERGSON, H. 1932. Les Deux Sources de la Morale et de la Religion. Paris : PUF. 1972. Mélanges. Paris: PUF.

.2009. Vie et œuvre de Ravaisson. In : La pensée et le mouvant. Éd. Critique Le Choc Bergson. Paris : PUF, p. 253-291.

MILET, J. 1970. Gabriel Tarde et la philosophie de l'histoire. Paris: Vrin. 
TARDE, G. 1993. Les lois de l'imitation. Paris : Editions Kimé. 1999a. L'opposition universelle, Vol. III. Paris: Les empêcheurs de penser en rond. 1999b. Monadologie et sociologie, Vol. I. Paris : Les empêcheurs de penser en rond. .1999c. La logique sociale, Vol. II. Paris : Les empêcheurs de penser en rond. 